\title{
Karl-Heinz Röhner Recht und soziale Wirklichkeit im Strafverfahren der DDR
}

\section{Instrumentalisierung des Verfabrens}

Wenngleich sich die Juristen der DDR auf dem Gebier des Strafverfahrensrechrs in der Vergangenheit nicht nur mit Normauslegung beschäftigten, wie man vielleichr aus der Tatsache schlußfolgern könnte, daß für die Erarbeitung des derzeit vorliegenden Kommentars zur Strafprozeßordnung der DDR fast zwei Jahrzehnte benötigt wurden', so kann andererseits aber auch nicht übersehen werden, daß sie sich einer wissenschaftlichen Durchóringung der sozialen Wirklichkeit des Strafverfahrens bisher - wenn überhaupt - nur punktuell zugewandt haben. Eine Soziologie des Strafverfahrens der DDR ist bisher weder geschrieben noch stehr sie in Aussicht.

Die Gründe dafür sind dabei keinesfalls nur darin zu suchen, daß Bemühungen um eine sozialwissenschaffliche Erforschung des Strafveríahrens leider allzu oft an den angeblichen Sicherheitsinteressen eines autoritären Staates scheiterten², sondern sie liegen nicht zuletzt auch in ciner vorwicgend inscrumentalistischen (funktionalistischen) Sichrweise auf das Strafverfahren begründet, infolge der das Surafverfahren im wesentlichen auf die Funkcion eines Inseruments zur Anrvendung des Sirafrechts beschränkt blieb. Leczrercs findet seinen Ausdruck sowohl in der gesctzlichen Bestimmung des $\$$ r Abs.: StPO, nach der das Strafverfahren der gerechicen Anwendung des Strafreches zu dienen habe, als auch im Lehrbuch Serafverfahrensrecht, in dem von einer dienenden Funkrion des Strafverfahreens gegenüber dem Strafrecht gesprochen wird ${ }^{3}$. Darüber hinaus herrscht diese Sichrweise auch bei nambaften Sirafverfahrensrechtswissenschaftlem der DDR vor. Das gilt beispielsweise für Beyer, wenn er das Strafverfahren als spezifische Form staatlicher Leirung zur Durchsetzung des Strafrechts begreift', aber ebenso für Luther, nach dessen Ansicht das Strafverfahren im Kem nStrafrechtsanwendungsverfahren «s ist. ${ }^{6}$

1 So crschien der crste Kommenlar zur Straprozeßordnuog der DDR vom 12. Januar 1y68 noch im Jahe ihres Inkrafteretens beum Statsverlag der DDR in Berlin. Eine zwette, völlig neubearbertetc Auflage dieses Kommentars erschien jedoch erse 1987 beim gleachen Verlag.

2 So scheiterten beispielsweise Versuche, cune Fihlureilsforschung zu betreiben, immer wieder an der ablchnenden Haltung zentraler Organc. Auch wctun diese Ablehnung nicht näher begründet wurdc, so dürfte dieser im wesentichen doch dic Befurchtung zugnunde gelegen haben, derarrge Forschungen könnten den Glauben an dic Unfehlbarkeıt des "sazıalistischen "Stastes erschüttern.

3 Vgl. Strafverfahrensrechr. Lehrbuch. 3., durchgesehene Aufl. Berlin: Staacsverlag der DDR 1987, S. 22.

4 Vgl. K. H. Beyer: Dic Funkeson des sozıalisuschen Sirufverfahtens im Kampl gegen die Kriminalitä und zu ihrer Vorbcugung bei der wciteren Gestaltung der encwickelien sozialisuschen Gescllschafe in der Deusschen Demokranischen Republik. Lespzig, Univ., Gesellschaftswiss. Fak., Diss. B. 1987, S. 361 ; derselbe: Die Funknon des sozialssuschen Siratverfahrens, seine Ziele und Aulgaben bei der weiteren schrittwessen Zuruckdrängung der Kriminalitat. Staat und Rechl 36 (1987) 7, S. $510 \mathrm{f}$

s Vgl. H. Luther: Gundlinien der Weiterentwicklung des Strafverlahrens und des Strafverfalirensrechis in der DDR. Stat und Recht 37 (1988) i2, S. 995 .

6 Fine ahnliche Sichtweise finda sich auch für das Sirafverfahrensrecht. So verstelien $H$. Luther und 
Eine solche vorrangig instrumentalistische Sichtweise auf das Strafverfahren verstellt indessen den Blick auf wesentliche soziale und recheliche Zusammenhänge? Sie verleiter nicht nur dazu, die Ziele, Mittel (Methoden) und Formen der prozessualen Tătigkeir vordergründig aus den Anforderungen der Anwendung des Strafrechts herzuleiten, d.h. recheliche Regelungsbedürfnisse und -notwendigkeiten aus dem Recht selbst zu erklären, sondern erweckt zugleich den Eindruck, als ließe sich die Effektivität des Strafverfahrens - bczogen auf dic Antvendung des Strafrechts allein schon (und beliebig oft) durch die Vervollkommnung verfahrensrechtlicher Bescimmungen erreichen. Insorvcit ist es dann auch niche verwunderlich, wenn im Lehrbuch Strafverfahrensrecht - quasi per definitionem - behauptet wird, daß das Strafverfahrensrecht dic effektivste Form für dic Anrvendung des Strafrechts bestimmt ${ }^{8}$, olne daß auch nur versuch wird, diese Behauptung durch entsprechende sozialwissenschaftliche Untersuchungsergebnisse zu stiitzen.

Darüber hinaus schließt eine instrumentalistische Sichtweisc immer dic Gefahr ein, daß Staat-Bürger-Beziehungen im Srrafverfahren zu ausschließlichen Leirungsbezichungen abqualifiziert werden. ${ }^{9}$ Der Einzelne wird zum Objekt staaticher (Strafverfolgungs-) Tätigkeit degradiert. Er ist dann lediglich Bezugspunkt, an dessen Adresse rechclich normiertc Verhaltensanforderungen übcrmittelt werden, welchen er sich in einem solchen Fall bestenfalls äußerlich anzupassen vermag. Das aber wiederum schließr die Gefahr ein, daß repressive Elemente und Momente der äußercn Disziplinierung im Serafverfahren die Oberhand gewinnen.

Vor dem Hintergrund eines solchen Instrumentalismus wird dann auch verständlich, wieso das Suratverfahren in der Vergangenheit niche nur gecignete Rabmenbedingungen für die Anrvendung eines in weiten Teilen repressiv-kontrollierenden Strafrechts bot, sondern auch selbst durcl, dic Praxis seiner Handhabung zum Instrument der Hintansetzung der Freiheitsinteressen des Bürgers zugunsten der Sicherheitsinteressen eines autoritären Staates werden konnte.

\section{Verdrängung von Widersprüchen und Konflikten}

Wie die bisherigen Ausführungen unschwer erkennen lassen, ist bei einer instrumentalistischen Sichtweise das Strafverfahren weder als soziale Existenzform noch als Austragungsform von Widersprüchen und Konflikten zwischen Individuum und Gesellschaft, Bürger und Staat begreifbar. Aber gerade das ist es, was die soziale Wirklichkcit des Strafverfahrens entscheidend prägt. Indem nämlich das Stralverfahren Austragungsform zwischen Individuum und Gesellschafo aufgebrochener Widersprüche und Konflikte im Zusammenhang mil der Prüfung und Feststellung der strafrechtlichen Verantwortlichkejt eines bestimmten Individuums ist, wird es zugleich selbst von diesen Widersprüchen und Konflikten durchdrungen und beeinflußt, wird es gewissermaßen selbst zu einem widersprüchlichen und konflikthaften Prozeß. Da aber die Austragung von Widersprüchen und Konflikzen im

L. Revier das Sirafverfaluensreche als nStrafreches-Anwindungsrechu*. Vel. H. Luther, L. Reueer: SerafprozeBrecluswissenschaft und Entwicklung des Sirafverfahrensrechts. Fruedrich-Schiller-Universıtät Jena, Forschungsergcbnisse, G/\$9/8, S. I.

7 Auslührlicher dazu: K.-H. Röhner: Funkuon und Wirksamken des soztalisuschen Siralverfahrens bet der Gestaltung des Verhälinisses von Individuum und Gescllschaft. Jena, Unv., Gesellschafisrviss. Fak., Diss. B, 1988, insbesondere S. 26 f.

8 Vgl. Siralverfiahrenstechi. Lehrbuch (Fn. 3), S.21.

9 Vgl. H.-J.Joseph: Einherelichkeır und Differenzıerung des Sirafprox.exses. Unuv., Jena, Gesellschafiswiss. Fak., Diss. A, 1982, S. 70 
Strafverfahren stets die Möglichkeit impliziert, diese Widersprüche und Konflikte in einer die Entwicklung der Gescllschaft und des Individuums fördernden Art und Weise zu lösen, aber auch ihre Lösung zu hemmen odcr gar ihre Lösung in eincr für die Gesellschaft oder die Enrwicklung des Individuums störenden oder schädlichen Richiung zu versuchen, bedarf auch das Strafverfahren norwendig rechrlicher Garancien gegenüber mißbräuchlicher Rechtsanwendung. Auch im Strafverfahren sind kulturgewordcne Werte wie Gerechtigkeit, Gleichheit, Freiheit, Humanismus, Sicherheir und andere von tragender Bedeutung.

Unter dem letztgenannten Aspekt enthalten Verfassung, Gerichtsverfassungsgesetz, Strafgesetzbuch und Strafprozeßordnung der DDR durchaus eine ganze Reihe rechtlicher Garanuien zur Verhinderung einer mißbräuchlichen Rechtsanwendung. ${ }^{10}$ Jedoch haben sich diese Garantien, wie die soziale Wirklichkeit des Strafverlabrens belegt, in der Vergangenheit nicht immer als hinreichend erwiesen, um unter allen Umständen ein faires Verfahren zu sichern. Die Gründe dafür sind vielfältiger Natur. Sie sind vor allem in dem bereits kritisierten Instrumentalismus zu suchen, der auch in der Justizpraxis vorherrschend war. In diesem Zusammenhang kann auch nicht übersehen werden, daß selbst dort, wo an sich hinreichende Garantien rechtlich gegeben waren, diesc aus Gründen der *Staatsräson * unterlaufen wurden und damit gegen grundlegende Bcstimmungen der Verfassung, des Gerichtsverfassungsgeserzes, des Strafgeserzbuches und der Srrafprozeßordnung verscoßen wurde.

\section{Paternalismus versus subjektive Rechte}

Fragt man nach den Ursachen, die zu einer ungenügenden Wirksamkeit dieser Rechtsgarantien in der Vergangenhcit führten, so finden wir sie - bei aller Vielfalc im Einzelfall - fetzclich in einer Denkart begründet, die vor dem gesamigesellschalchchen Hintergrund ciner paternalistischen, dic Individualitäl des Einzelnen und seine Souveränität verdrängenden Bevormundung durch den Staat die Subjektposicion des tatverdächtigen Individuums" alicnfalls als etwas Sekundäres betrachtete. " Damit aber erhieit im Grunde trotz vieler ehrlichor und gutgemeincer Bemüluungen und Sorgen um die Resozialisierung des Individuums das repressive Moment im Strafverfahren das Übergewicht.

Eine Lehre aus der Vergangenheit muß also zwangsläufig darin gesehen werden, die Subjektposition des tatverdächrigen Individuums stärker auszubatten und zu sanktionicren. Das tatverdächige Individuum muß aus der Sicht seiner sozialen Aktivirät als ciner Scite jener Wechselwirkung zwischen Individuum und Gesellschaft, in der sich der Lebensprozeß vollzieht, sowie eines sozial agierenden, das Strafverfah-

10 Hicr sollen nur emige von ihnen genanne werden: z. B. das Verboe von Ausnahmegerichten (Ara. ro Abs. 2 Verfassung): dic Unabhängigkeul der Richter (Ar. 96 Abs. 1 Verfassung, \$ s Abs. 2 GVG. \$9 $\Lambda$ bs. 1 SiPO): dic Sicherung der Unvorengenommenhell der Richeer ( 7 GVG, 8 Abs. I, 9 Abs. 1 , 156(f. SePO); die Einschränkung der Rochte der Bürger im Stenfierfahren nur insopres, wie dies

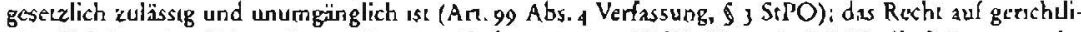
ches Gchör und auf Vercèdigung (An. 102 Verfassung, $\$$ is GVG, $\$$ is, 61 StPO); dic Präsumuon der Unschuld und der Grundsaiz - in dubio pro reo. (Ar. 4 SiGB, $\$ 6 \wedge$ bs. 2 StPO); die Untersagung aller die frete Willensentschließung der Veriahrensbeceiligan durch Zwang beeinfussenden Vernehmungsmethoden $(\$ 243 \mathrm{StGB})$; die Androhung strafrechtlicher Verantworilichket bet Rechisbeugung $(\$ 244$ $\mathrm{S}(\mathrm{GB})$.

11 Der Begrifl des caeverdachugen Individuums ivird hier und un weteren im Sinne concs Oberbegrills fur das bescliuldige und/oder angeklagte Individuum verwendet.

I2 Vgl. G. Hancy: Staat - Gexellschaft-Individuum. Ein Diskussionsbetrag. Staar und Rechr $3^{8}$ (1989) I 2 , S. $971-9$ SO. 
ren mitgestaitenden Subjekts begriffen werden. Das aber hat Konsequenzen für die

Durchführung des Strafverfahrens, die bisher weder praktisch noch theorecisch hinreichend ausgelotet sind.

Wird nämlich die akrive Einbeziehung des tatverdächrigen Individuums in das Strafver\{ahren als notwendiger Ausdruck seiner staatsbürgerlichen und Rechrsstellung im Strafverfahren verstanden, so sind ihm im Strafverfahren unter Berücksichtigung seiner individuellen Möglichkeiten und Fähigkeiten Handlungsräume zu eröffnen, die íhm eine prakrische Aneignung der vermitrelten Normeinsichten und Handlungserfordernisse emöglichen. Das tatverdächtige Individuum muß auch im Strafverfahren Subjekt aktiver - wenn auch spczifischer - Täcigkeirsbeziehungen werden können. Das herkömmliche repressive Moment des Strafverlahrens ist zunehmend durch eine die Eigenverantwortung und Selbstbestimmungsfähigkeit des tatverdächtigen Individuums deutlicher herausstellende Gestaltung des Strafverfahrens zurückzudrängen. Damit aber sind unter dem Blickwinkel der Subjektposition des tatverdächtigen Individuums allgemcin höhere Ansprüche an die Kultur des Verfahrens gestellt.

\section{Kontradiktorische Gestaltung des Verfabrens}

Ein höherer Anspruch an die Verfahrenskultur in der DDR schließt zwangsläufig die weitere Ausprägung des kontradiktorischen ("streitigen «) Charakrers des Strafverfahrens ein, eine Forderung übrigens, die in der Vergangenheit auch unter den Strafveriahrensrechuswissenschafelern der DDR nicht unumstritten war. Wurde Kontradiktion im Straiverfahren zunächst mit dem Parteiprinzip gleichgesctzt und abgelehnt's, so hat diese in den zurückliegenden Jahren in der wissenschaftlichen

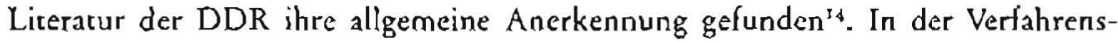
wirklichkeit ist man jedoch nach wie vor weit von einer koncradikcorischen Gesta\}rung des Verfahrens entfernt. Noch zu oft isc z. B. in der gerichtlichen Haupeverhanḋlung der Vorsitzende des Gerichts so dominant, daß der Eindruck entsceht, Staatsanwalt, Verteidiger - sowcit überhaupt einer mitwirkt - und andere Verfahrensbereiligte seien nur Statisten. " Außerdem setzt Kontradiktion im Strafver\{ahren notwendigerweise eine aktive Rolle des tarverdächtigen Individuums im Ringen um die Wahrheit voraus, eine Rolle indessen, die maßgebliclı vom Rang abhängt, dem man dem Reche des racverdächcigen Indiviơuums auf Verteidigung im Strafverfahren einräumt. Hier aber ergeben sich mit Blick auf Verfahrensrech und Verfahrenswirklichkeit cine Reihe von Problemen. So steht zunächst die Unwissenheit des tatverdächtigen Individuums in Fragen des materiellen und Prozeßrechts einer aktiven Rolle im Strafverfahren entgegen. Zwar verpflichter die Strafprozeßordnung die Strafrechtspflegeorgane, das catverdüchrige Individuum im jerveiligen Verfah-

13 Vgl. L. Rcutcr: Besprechung von "Das Recht des Beschuldigten und Angcklagecn auf Vereidigung im sozisisustischen Straiprozeßa. Neuc Just1\% 39 (1985) 7. S. 300 .

14 Vgl. H. Luther: Zur Polemik gegen die These vom koneradiktorischen Charakeer des Strafuerfahrens der DDR. In: Stralprozessuale Secllung und Funkuon des Stastsanwalts um Strafverfahren der DDR. Univ. Jena. 198 5. S. 143-148: M. Hirschfelder: Zum Reche auf Verteidigung und zum Pareiprınzip. In: Zur Entrvicklung des soziaksuschen Stralverfahrensrechts der DDR - Wesenszüge, Probleme, Perspekuven. Leipasg 1989, S. 154-158: L. Reuter; Kontradiktion in der gerichtlichen Beweisaulnahme. In: Genchtli-

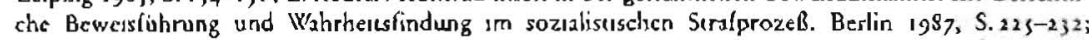
M. Hirschfelder: Das Recht aul Vereedigung un Stralverfahren der Deutschen Demokrauschen Republik. Unıv., Bcrlin. Gesellschafeswiss. Fak., Diss. B, 1989, S.61f.

is Vgl. L. Reuter: Kontradikion in der gerichtichen Bewelsauinahme (Fn. 14), S.22sf. 
rensscadium über seine Rechre zu belehren, doch geschieht das in der Praxis zumcist so pauschal, daß daraus kaum Akrivitäten des tarverdäclarigen Individuums zu erwachsen vermögen. Zudem steht dem tatvcrdächtigen Individuum auch kein Recht zur Akteneinsicht zu. Es ist folglich auch hier auf das angewiesen, was ihm die Strafrechtspflegeorgane in Realisierung ihrer gesetzlichen Verpflichtung über die vorliegenden Beweismittel mitteilen. Da eine solche Information in der Praxis meist auch nur oberflächlich erfolgt, ist sie für eine aktive Selbstverteidigung ebenfalls wenig hilfreich. Andererseits kann in Fällen der Gefährdung der Sicherlseit des Sraaces oder im Interesse der Geheimhalnung bescimmter Tatsachen auf eine Aushändigung von Anklageschrift, Eröffnungsbeschluß und Urteil (Beschiuß) an das tarverdächrige Individuum verzichter werden. ${ }^{16} \mathrm{Ihm}$ müssen diese Prozeßdokumence lediglich zur Kenntnis gebracht werden. Eine solche geseczlich legitimierte Praxis läuft als Ausdruck übcrzogener Sicherheitsinteressen des Staates ebenfalls dem Interesse des betroffenen Individuums an einer aktiven Wahrnahme seines Rechts auf Verteidigung zuwider.

Mit Blick auf diese - hier nur im groben skizzierren - gesetzlichen und carsächlichen Einschränkungen für eine akrive Selbstverteidigung des tatverdächtigen Individuums gewinnt die Möglichkeit seiner Verteidigung durch einen Rechtsanwalt um so größere Bedeutung. Aus diesem Grund wurde in der DDR bereils wiederholt vorgeschlagen, dem tatverdächtigen Individuum in jeder Verhandlung vor einem staatlichen Gericht einen Rechtsanwalt als Verteidiger zur Seite zu stellen. ${ }^{7}$ Allerdings ist eine Verwirklichung dieses Vorschlags auf Grund der geringen Zahl in der DDR zugelassener Rechtsanwälte derzeit völlig unreal. Daneben wirken sich aber auch gesetzlich vorgeschene Beschränkungen nachteilig auf die Verteidigung durch cinen Rechtsanwalt aus. So kann beispieisweisc der Staatsannalt dem Verteidiger die Aktencinsicht vor Abschluß der Ermittlungen verweigern, wenn die Untersuchungen dadurch gefährdet sind. Unrer denselben Voraussetzungen kann der Verıeidiger auch von der Teilnahme an Beweiserhebungen im Ermittlungsverfahren ausgeschlossen werden. Auch wenn diese Regelungen in der Praxis als Ausnahmeregein verstanden werden sollten, so blciben sie lecztlich doch immer Ausdruck überzogener Sicherheitsinteressen des Staates. Zugleich kann und darf in dicsem Zusammenhang auch nichı übersehen werden, daß in der Vergangenbeit Rechtsanwälte wegen ihrer engagierten Verteidigung von Bürgern, vor allem solcher, die im Verdacht standen, Straftacen gegen die DDR begangen zu haben, gemaßregelt wurden oder in bestimmten Fällen sogar ihre Zulassung als Anvvalt verloren. ${ }^{18}$

Höhere Ansprüche an die Kultur des Verfahrens müssen folglich notwendigerweise Überlegungen zum weitcren Ausbau des Rechts auf Verteidigung einschließen. Dabei kommt es vor allem darauf an, vorhandene Einschränkungen des Rechts auf Verceidigung, soweit sie Ausdruck ungcrechtfertigter Sicherheitsinteressen des Staates sind, aufzuhcben und darüber hinaus weitere Garanticn für eine akcive Wahrnabme dieses Rechts geserzlich zu verankern.

$16 \mathrm{Vgl}$ dic Regclungen der $\$ \mathrm{I}_{4}$ Abs.5, $203 \mathrm{Abs}, 3 \mathrm{StPO}$

$17 \mathrm{Vgl}$. H. Luther. F. Wolll: Das Recht auf Veriesdigung in sozialiseschen Strafueríahren. Staal und Recht 27 (1978) 2, S. 449; M. Hirschíclder: Das Rechi auf Verteidigung im Seralverfaliren der Deutschen Domokrauschen Republik (Fn. 14), S. 1 jo.

18 Deraruge Prakuken blieben in der Vergangenherr der Óffendichkeit weugehend verborgen. Sie werden erst heuce im Zusammenhang nut der Rehabiliucrung der betrofienen Rechesanwalte bekanne. So war beispielsweise 1976 dem Rechesanwait Dr. Gouz Berger Im Zusammenhang mit der Übernahme der Vericidigung von Prof. Dr. Robert Havemann dic Zulassung entzogen worden. Vgt, "Rechisanwale Dr. Berger wurde reliabilitıcre.. Neucs Deutschland vom 22.11.19\$9, S. 2. 
Höhere Ansprüchc sind aus der Sicht der Kuitur des Verfahrens künfrig auch an die Achtung der Präsumtion der Unschuld des tatverdächrigen Individuums zu stellen. Dies gilt nicht etwa nur deshalb, wcil in der Vergangenheit in sogenannten Schauprozessen gegen angebliche Staatsfeinde für jedermann offensichtlich eine * Vorvcrurreilung " der Betroffenen bcreits statigefunden hatte', sondern vor allem mir Blick auf den auch im Strafverfahren der DDR gelcenden Resozialisierungsgedanken, der allzu schnell dazu verleitet, auf das tatverdächtige Individuum in einer Weise einzuwirken, die einer vorweggenommenen Schuldfeststellung gleichkommt. Hier setzt der Grundsatz der Präsumuion der Unschuld strikt zu achtende Grenzen. Er verbietet jegliche vom Resozialisicrungsgedanken getragene fremderzieherische Einwirkung auf das tatverdächrige Individuum.

Dennoch schließt die Achtung der Präsumtion der Unschuld eine am Resozialisierungsgedanken orientierte Gestaltung des Strafverfahrens nicht aus. Allerdings darf eine solche Gestaltung nichs auf eine fremderzicherische Einwirkung auf das tatverdächrige Individuum durch bestimme prozessuale Handiungen oder Maßnahmen anderer Verfahrensbeteiligter hinauslaufen. Sie ist vielmehr einer persönlichkeitsfördernden Gestaltung und persönlichkeirsgerechten Individualisierung der prozessualen Tärigkeit selbst gleichzusetzen, die die eıgene Akcivität des catverdächtigen Individuums fördert und das Ingangsetzen bzw. -halten scines eigenaktiven selbstkritischen Erkenntnis“ und Veränderungsprozesses unterstürzt. Hierzu gehört aber auch, daß durch die Organe der Strafrechrspflege Erscheinungen der Befangenheit, Voreingenommenheit und Ablehnung gegenüber dem tarverdächtigen Individuum von seiten anderer Verfahrensbeteiligter entgegengewirkt wird, um insoweir keine "Vorverurteilung zuzulassen.

Kehrt man nach diesen Ausführungen über die Kultur des Verfahrens zum Ausgangspunki der Betrachtung zurück, so wird erkennbar, daß zwischen Recht und sozialer Wirklichkeit im Strafverfahren eine Spannung besteht, die sich indessen nicht einseitig vom Recht zur sozialen Wirklichkeit hin erstreckt, sondern umgekehrt auch von der sozialen Wirklichkejt zum Recht. Das Spannungsverhältnis von Reche und sozialer Wirklichkcit im Strafverfahren wäre folglich nichr schon allein dadurch hin reichend charakterisiert, daß man eine ungenügende Umseczung rechclicher Anweisungen in die soziale Wirklichkeit des Strafverfahrens konstaciert, die sich nach Roxin in der einfachsten Form dort zeigt, wo das wirkliche Geschehen einer Normanweisung zuwiderläu\{t « $^{20}$. Notwcndig ist vielmehr auch eine Untersuchung, inwieweit die im Recht enchaitene Anwcisung überhaupt den in der sozialen Wirklichkeit angelegten Möglichkeiten und Bedingungen zur Austragung von Widersprüchen und Konflikien zwischen Individuum und Gesellschaft angemessen (adäquar) ist. Es darf keinesfalls überschen werden, daß diesc in der sozialen Wirklichkeit angelegten Möglichkeiten und Bedingungen grundsätzlich den Rahmen (die Grenzen) abstecken, in dem (in denen) sich die (rechtlichc) Austragung von Widersprüchen und Konflikten im Strafverfahren bewegen kann. In der Art und Weise, wie daher die Gescllschafr im Strafverfahren die Widcrsprüche und Konflikte des Einzehnen mit ihr zu bewälrigen bemüht ist, widerspiegelt sich somit

19 Der "Fall Jankax mag haer als traunges Beispiel dienen. Ausführlichcr: «Ungeserzliches Urtal im Verfahren gegen Waleer Janka, Gustav Juse, Heinz, Zöger und Richand Wolf aufgehobin (Urecil, Beschluß über den Eneschädigungsanspnich und Plädoyer von Rechtsanwalt Dr. F Wallf um Kassaaonsverfaheen vor dem Obersien Genche) a, Ncue Jusuz 44 (1990) 2, S. 50-g1.

$20 \mathrm{C}$. Roxin: Reche und soziale Wirklichkeir im Sirafveriahren. In: Kriminologie und Strafverfalsren. Seungan: Enke 1976. S. 12. 
auch das jeweils historisch errcichte Niveau der Kultur der Gesellschaft im Umgang mit ihren Individuen und damit die reale Stellung des Einzelnen in ihr.

Geht man von dem Gesagten aus, so stellt sich ein spezifischer Zusammenhang zwischen Strafverlahren und gesamtgesellschaftlicher Entwicklung in der DDR her. Es wird erkennbar, daß der Ausbau der Subjcktposition des tazverdächtigen Individuums im Strafverfahren norwendigcrweise einen Umgang der Gesellschaft mit iluren Individuen voraussetzt, der auf breitester demokratischer Grundlage von dem Bemühen der Gesellschaft um die freie Individualitärsentraltung des Einzelnen getragen wird und damit eine paternalistische, die Individualität des Einzelnen und seine Souveräni̊ät beschränkende Bevormundung durch den Staat ausschließt. Die ersten Schritte hierzu sind bereits getan, wie die gegenwärtige gesellschaftliche Entwicklung in der DDR zcigt. 